\section{Case Reports in Ophthalmology}

Case Rep Ophthalmol 2018;9:87-91

\title{
Optical Coherence Tomography Angiography of Foveal Neovascularization in Diabetic Retinopathy
}

\author{
Konstantinos Andreanos Tryfon Rotsos George Kymionis \\ Chryssanthi Koutsandrea Athanasios Kotsolis Ioannis Ladas
}

1st Department of Ophthalmology, University of Athens, Georgios Gennimatas General Hospital, Athens, Greece

\section{Keywords}

Optical coherence tomography angiography · Diabetic retinopathy · Foveal neovascularization

\begin{abstract}
Purpose: To report a case of foveal neovascularization in a patient with proliferative diabetic retinopathy as seen on optical coherence tomography angiography (OCT-A). Methods: Multimodal imaging was used for diagnostic investigation. Patient: A 61-year-old male with a 16-year history of insulin-dependent diabetes mellitus was referred to our medical retina department for examination and management. Meticulous fundus examination and multimodal imaging revealed proliferative diabetic retinopathy lesions, including neovascularization located in the foveal area. Results: OCT-A allowed us to detect the neovascular lesion, confirm that it originated from perifoveal capillaries, estimate its retinal depth, and evaluate the vessel blood flow in multiple layers. Conclusion: To the best of our knowledge this is the first report of OCT-A imaging of foveal neovascularization in diabetic retinopathy. OCT-A is a very useful examination for the diagnostic investigation of patients with diabetic retinopathy.




\section{Case Reports in Ophthalmology}

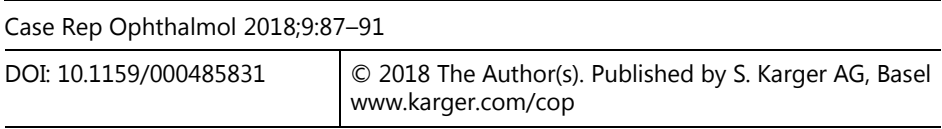

Andreanos et al.: Optical Coherence Tomography Angiography of Foveal Neovascularization in Diabetic Retinopathy

\section{Introduction}

Retinal neovascularization is the hallmark of proliferative diabetic retinopathy and it is triggered by decreased oxygen levels as a result of retinal capillary nonperfusion. It is most commonly located at or near the optic nerve head, or at the borders of perfused and nonperfused retina, sparing the foveal area. In the literature, there have been few reports of retinal neovascularization growing in the foveal area [1-4]. Herein, we present a case of foveal neovascularization (NVF) as seen on optical coherence tomography angiography (OCT-A) in a patient with proliferative diabetic retinopathy (Fig. 1).

\section{Case Presentation}

A 61-year-old male with a 16-year history of insulin-dependent diabetes mellitus was referred to our medical retina department for examination and management. Best corrected visual acuity was $6 / 60$ in his right eye and counting fingers in the left. Intraocular pressure and the anterior chamber were normal in both eyes. Meticulous examination of the iris did not reveal any sign of iris neovascularization.

Fundus examination revealed multiple microaneurysms, dot and blot hemorrhages, and several hard exudates in the macula of both eyes (Fig. 1e). Fluorescein angiography showed late leakage of the dye due to the existing microaneurysms and extensive capillary nonperfusion areas in the fundus, but no sign of leakage due to retinal neovascularization on the optic disc or along the vascular arcades (Fig. 2). However, typical leakage of the dye due to neovascularization was observed in the fovea of the left eye. Swept-source OCT scans demonstrated retinal edema, an epiretinal membrane, and multiple hyperreflective signals corresponding to hard exudates. NVF was seen as a uniform mass of moderate reflectance underneath the internal limiting membrane.

OCT-A using the DRI OCT Triton system (Topcon Co., Tokyo, Japan) was performed. OCT angiograms were acquired at a $3 \times 3 \mathrm{~mm}$ zone centered on the location where the NVF was localized. Due to patient's poor visual acuity and eye motion the image generated demonstrated several displacement and white line artefacts (Fig. 1a, b) [5]. Image processing was performed in order to correct these artefacts (Fig. 3). Furthermore, a chromatic blood flow density map was created based on the superficial slab. NVF was indeed imaged in detail in the superficial plexus slab and it originated from the perifoveal microvascular plexus. Chromatic blood flow density maps of the foveal zone show reduced blood flow in the vascular plexuses of all levels.

\section{Discussion}

Neovascularization in proliferative diabetic retinopathy often occurs at or near the optic disc or within an area of 3 disc diameters distally by the major retinal vascular arcades. As a rule, the fovea is spared from neovascularization because the underlying choriocapillaris is densely vascular and oxygen concentration is maintained even in the presence of perifoveal retinal capillary dropout. Nevertheless, choroidal blood flow and choroidal volume have been shown to be significantly reduced in patients with type 2 diabetes compared to normal subjects, especially in patients with macular edema [6]. In such a hypoxic foveal microenvironment, NVF can develop, as seen in similar previous reports [1-4]. 
Andreanos et al.: Optical Coherence Tomography Angiography of Foveal Neovascularization in Diabetic Retinopathy

We report a case of NVF in a patient with insulin-dependent diabetes, as seen in OCT-A. Typical leakage due to neovascularization was indeed detected on fluorescein angiography in the foveal area, but visualization of the lesion was poor. OCT-A allowed us to detect the characteristic neovascular pattern in the superficial vascular plexus which could be clearly seen originating from perifoveal capillaries, while deeper angiograms seemed to be neovascularization free. Chromatic blood flow density maps of the foveal zone confirmed reduced blood flow in all vascular plexuses of all levels, which seems to be the main cause of the development of NVF.

In conclusion, OCT-A is a relatively new modality that allowed us to detect the foveal neovascular lesion in diabetic retinopathy, confirm that the lesion originates from perifoveal superficial capillaries, estimate its retinal depth, and evaluate the vessel blood flow in multiple layers. The use of OCT-A imaging gives us much new information which will help us understand the pathophysiology of several macular pathologies and detect their clinical manifestations.

\section{Summary Statement}

We report a case of foveal retinal neovascularization, a rare clinical manifestation of diabetic retinopathy. We describe OCT-A findings of the lesion and highlight the usefulness of this novel modality.

\section{Statement of Ethics}

The patient involved provided a written informed consent, in accordance with the tenets of the Declaration of Helsinki, to having their medical data used for research purposes.

\section{Disclosure Statement}

The authors report no conflicts of interest. The authors alone are responsible for the content and preparation of this manuscript.

\section{References}

1 Joondeph BC, Joondeph HC, Flood TP: Foveal neovascularization in diabetic retinopathy. Arch Ophthalmol 1987;105:1672-1675.

-2 Finkelstein D, Patz A, Fine SL, Rice TA, Murphy RP: Abortive foveal retinal neovascularization in diabetic retinopathy. Retina 1981;1:62-66.

-3 Rajagopal J, Kamath AG, Kamath GG, Solanki N: Foveal neovascularisation in diabetic retinopathy: case report and review of literature. Int Ophthalmol 2010;30:311-314.

-4 Kurz PA, Nguyan H, Cooney MJ: Bilateral foveal neovascularization in a patient with insulin-dependent diabetes mellitus. Arch Ophthalmol 2003;121:1656-1657.

-5 Spaide RF, Fujimoto JG, Waheed NK: Image artifacts in optical coherence angiography. Retina 2015;35:2163-2180.

-6 Nagaoka T, Kitaya N, Sugawara R, Yokota H, Mori F, Hikichi T, Fujio N, Yoshida A: Alteration of choroidal circulation in the foveal region in patients with type 2 diabetes. Br J Ophthalmol 2004;88:1060-1063. 


\section{Case Reports in Ophthalmology}

\begin{tabular}{l|l}
\hline Case Rep Ophthalmol 2018;9:87-91 \\
\hline DOI: 10.1159/000485831 & $\begin{array}{l}\text { C 2018 The Author(s). Published by S. Karger AG, Basel } \\
\text { www.karger.com/cop }\end{array}$ \\
\hline
\end{tabular}

Andreanos et al.: Optical Coherence Tomography Angiography of Foveal Neovascularization in Diabetic Retinopathy
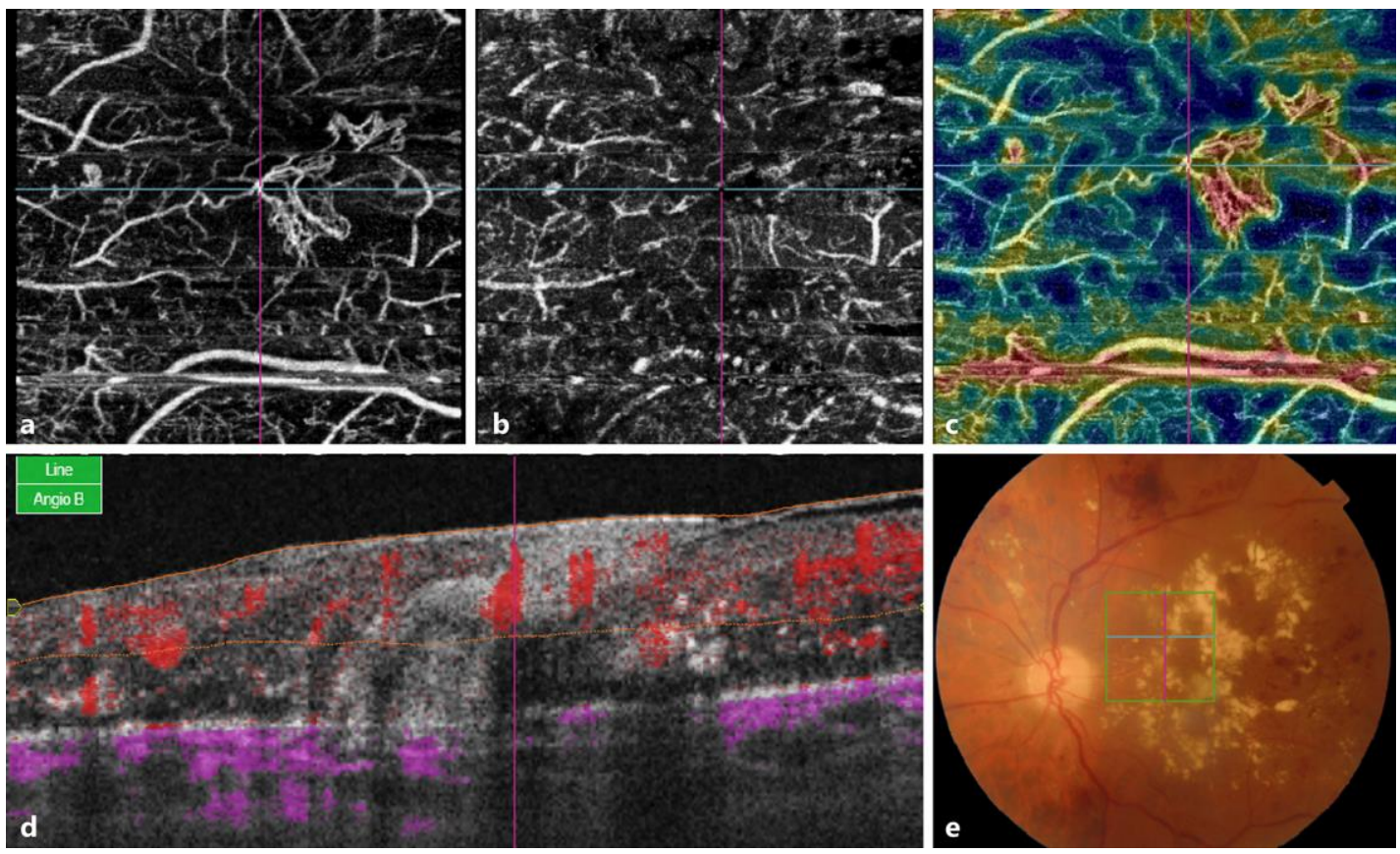

Fig. 1. OCT-A imaging of foveal neovascularization (NVF). a Superficial plexus, where the lesion is located. b Deep retina plexus, which shows no sign of neovascularization. c Blood flow topographic map. $\mathbf{d}$ OCT Bscan, showing NVF as a uniform mass of moderate reflectance underneath the internal limiting membrane. e Color fundus image, showing multiple microaneurysms, dot and blot hemorrhages, and several hard exudates.
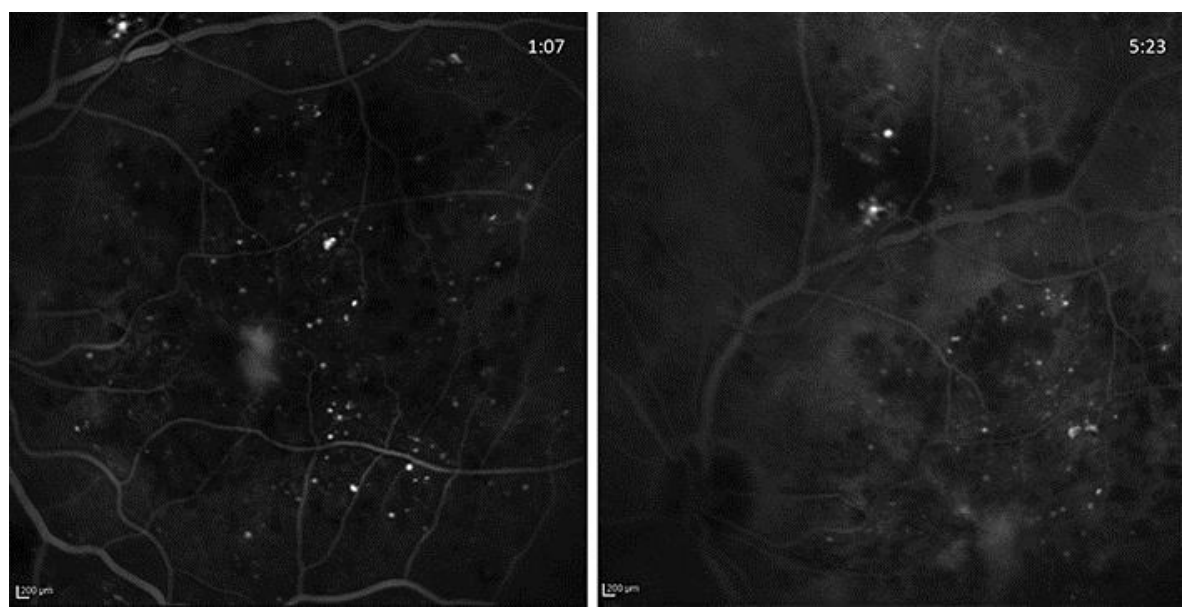

Fig. 2. Fluorescein angiography reveals typical leakage of the dye due to retinal neovascularization both in early (left) and late phases (right). 


\section{Case Reports in Ophthalmology}

\begin{tabular}{l|l}
\hline Case Rep Ophthalmol 2018;9:87-91 \\
\hline DOI: 10.1159/000485831 & $\begin{array}{l}\text { C 2018 The Author(s). Published by S. Karger AG, Basel } \\
\text { www.karger.com/cop }\end{array}$ \\
\hline
\end{tabular}

Andreanos et al.: Optical Coherence Tomography Angiography of Foveal Neovascularization in Diabetic Retinopathy

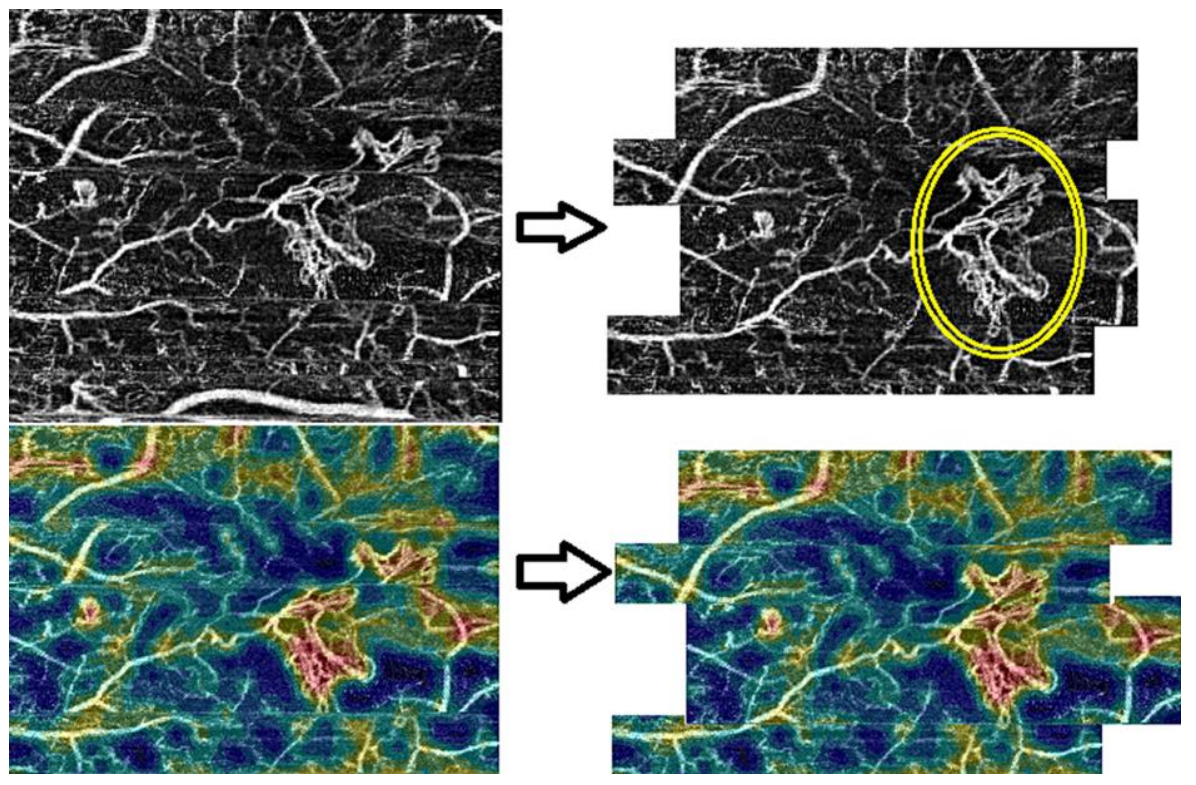

Fig. 3. Processed images where displacement artefacts have been corrected. Superficial plexus slab (upper row), where it is evident that the neovascularization (inside yellow oval) emanates from superficial perifoveal capillaries. Blood flow topographic maps (lower row) indicating low blood flow in the perifoveal area and high blood flow in the neovascular lesion. 